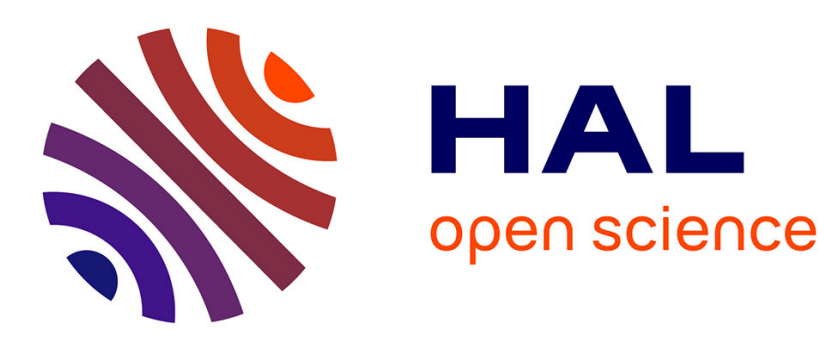

\title{
On a Computational Strategy with Time-Space Homogenization for Heterogeneous Materials
}

\author{
Anthony Nouy, Pierre Ladevèze
}

\section{To cite this version:}

Anthony Nouy, Pierre Ladevèze. On a Computational Strategy with Time-Space Homogenization for Heterogeneous Materials. Journal of the Mechanical Behavior of Materials, 2009, 19 (2-3), pp.115-124. 10.1515/JMBM.2009.19.2-3.151 . hal-02163502

\section{HAL Id: hal-02163502 https://hal.science/hal-02163502}

Submitted on 2 Oct 2020

HAL is a multi-disciplinary open access archive for the deposit and dissemination of scientific research documents, whether they are published or not. The documents may come from teaching and research institutions in France or abroad, or from public or private research centers.
L'archive ouverte pluridisciplinaire HAL, est destinée au dépôt et à la diffusion de documents scientifiques de niveau recherche, publiés ou non, émanant des établissements d'enseignement et de recherche français ou étrangers, des laboratoires publics ou privés. 


\title{
On a Computational Strategy with Time-Space Homogenization for Heterogeneous Materials
}

\author{
Anthony Nouy \\ LMT-Cachan (ENS Cachan / CNRS / Université Paris VI) \\ 61 Avenue du Président Wilson, F-94235 Cachan CEDEX, France \\ Pierre Ladevèze \\ LMT-Cachan (ENS Cachan / CNRS / Université Paris VI) \\ 61 Avenue du Président Wilson, F-94235 Cachan CEDEX, France \\ pierre.ladeveze@lmt.ens-cachan.fr
}

\begin{abstract}
SUMMARY
A new multiscale computational strategy was recently proposed for the analysis of structures described both on a fine space scale and a fine time scale. This strategy, which involves homogenization in space as well as in time, could replace in several domains of application the standard homogenization techniques, which are generally limited to the space domain and present some drawbacks. It is an iterative strategy which calls for the resolution of problems on both a micro (fine) scale and a macro (homogenized) scale. Here, we review the bases of this approach and present improved approximation techniques to solve the micro and macro problems.
\end{abstract}

\section{INTRODUCTION}

When a precise solution is required in the analysis of heterogeneous structures, such as reinforced or composite structures, the calculation must be performed on a fine discretization of the structure (on the micro-level). Since the constituents often have very different mechanical characteristics, the resulting structure is highly heterogeneous and the local solution displays phenomena with a short length of variation. This type of situation leads to problems with very large number of degrees of freedom and the calculation cost is generally prohibitive if one uses classical FE codes. Our objective is to reduce the calculation cost drastically while, at the same time, trying to improve the robustness.

The theory of periodic media homogenization initiated by Sanchez-Palencia [1] is one of the possible strategies to achieve this goal, especially for linear problems. Modified versions have been developed to 
handle nonlinear problems [2-5]. However, all these theories are valid only for small ratios between the small scales and the large scales. Moreover, a specific treatment is required at the boundaries, where the material cannot be homogenized.

An answer to this challenge is a new micro-macro computational strategy [6-9] which includes an homogenization technique in both space and time while avoiding the drawbacks of the classical homogenization theory. It is iterative and works over the entire space-time domain. Homogenization is automatically performed along iterations. This approach can be seen also as an alternative to classical homogenization theories. Here, this strategy is detailed for (visco)plastic materials and optional unilateral contact with or without friction.

The first feature of the method consists of partitioning the space-time domain. The structure is an assembly of substructures and interfaces. Each component has its own variables and its own equations. The junction between the "macro" and the "micro" scales takes place only at the interfaces. "Macro" and "micro" quantities are sort of mean values over both space and time. The resulting structure can be interpreted as a Cosserat-like medium.

The method's second feature is the use of the so-called LATIN method, a non-incremental iterative computational strategy applied over the entire time interval being studied [10]. At each iteration, one must solve a "macro" problem, defined on the entire structure and the entire time interval, along with a family of independent linear problems each defined on a composite cell and its boundary. These are "micro" problems, unlike the "macro" problem which corresponds to the entire homogenized structure both in time and in space.

Here, we focus first on the basic aspects of this approach and in particular on the homogenization procedure in space and in time. Further works are also presented, which help reduce the computation cost. A first improvement consists of intoducing a third scale to build an approximation of the "macro" problem. Another improvement concerns the resolution over the space-time domain of the "micro" problems for which a "radial-type" approximation [10] is introduced.

Several numerical examples illustrate the possibilities of the approach presented.

\section{THE REFERENCE PROBLEM}

We consider a standard boundary value problem under the assumptions of small perturbations and isothermal, quasi-static state. The inelastic strain $\varepsilon_{p}$ is considered separately from the other internal variables, denoted $X$. The conjugate variable of $X$ is $Y$; thus, the dissipation rate is $\left.T \cdot \sigma \dot{\varepsilon}_{p}\right]-Y \cdot \dot{X}$, where $\sigma$ denotes the stress field. From the free energy, under usual decoupling assumptions, we obtain a "normal" formulation [10] of the state equations.

The first step of the multiscale computational strategy consists of describing the structure as an assembly of simple components: substructures and interfaces [10]. Each component has its own variables and 
equations. A substructure $\Omega_{E}, E \in \mathbf{E}$, is subjected to the action of its environment (the neighboring interfaces) defined by a force distribution $F_{E}$ and a velocity distribution $\dot{w}_{\mathfrak{a}}$ on its boundary $\partial \Omega_{\varepsilon}$. An interface $\Gamma_{E E \mathcal{O}}$ between two substructures $E$ and $E$ transfers both the velocity and the force distributions: $\dot{\mathrm{W}}_{,}, \dot{\mathrm{w}}$. and $F_{E}, F_{E O}$. The set of unknowns on a substructure and its boundary is denoted $s_{E}$. $\mathbf{s}_{E}=\left(\varepsilon_{E}, X_{E}, \sigma_{E}, Y_{E}\right)$ is said E-admissible if it verifies the equilibrium equations, the compatibility equations, the state equations and the initial conditions.

The reference problem can be reformulated as follows: Find $s=\bigcup_{E \in E} s_{E}$ which verifies the Eadmissibility of $s_{E}$, the constitutive relation describing the state evolution and the behavior at the interfaces.

\section{DESCRIPTION OF QUANTITIES ON THE MACROSCALE AND ON THE MICROSCALE IN THE SPACE-TIME DOMAIN}

The distinction between the micro and macro levels is made only at the interfaces. For space, the macro scale is defined by the characteristic length of the interfaces, which is a priori much larger than the discretization scale. For time, the macro scale is associated with a coarse partitioning of the time interval $\tau_{h \mathcal{O}}=\left\{t_{0}^{\Theta}=0, t_{1}^{\odot}, \ldots, t_{N}^{\Theta}=T\right\}$ being studied, included in a finer partitioning $\tau_{h}$. For example, one could take, on the space level, affine functions on an interface for macro forces and displacements $W^{M}$ and $E^{M}$ and, on the time level, piecewise polynomial functions of degree $p$ for the grid $\tau_{h 0}$ : the corresponding space is denoted by $\mathbf{Z}_{\infty}^{[0, T]}$. The expression of work is taken in the sense of the Galerkin discontinuous method [11]. The micro parts are $E^{m}=E-E^{M}$ and $W^{m}=\underline{W}-\underline{W}^{M}$. "Macro" and "micro" parts of forces and displacements on the interface are uniquely defined by the following decoupling property

$$
\int_{\Gamma_{E E O \times[0, T]}} \underline{F} \cdot \underline{W} d S d t=\int_{\Gamma_{\ell: E O \times[0, T]}}\left(\underline{F}^{M} \cdot \underline{W}^{M}+\underline{F}^{m} \cdot \underline{W}^{m}\right) d S d t
$$

The rationale adopted for the definition of the macro quantities is physically sound: these quantities are mean values in space as well as in time. More precisely, they are the best approximations in the sense of the work bilinear form.

Another important feature of the multiscale computational strategy presented here is that the transmission conditions at the interfaces are partially verified a priori. The macro forces are required to systematically verify the transmission conditions, including the boundary conditions. These conditions remain unchanged under unilateral contact. 


\section{MULTISCALE COMPUTATIONAL STRATEGY WITH SPACE AND TIME HOMOGENIZATION}

\section{口 Principle}

Partial verification a priori of the transmission conditions at the interfaces leads to the following reformulation of the reference problem: Find $s=\bigcup_{E \in E} s_{E}$ which verifies:
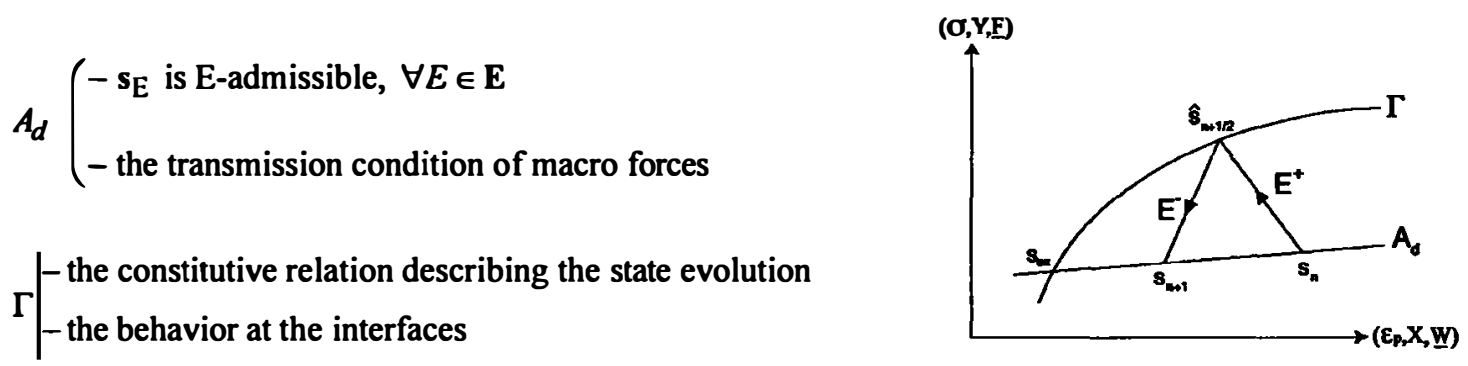

Fig. 1: Scheme of one iteration

$\Gamma$ represents a set of (possibly nonlinear) equations which are local in the space and time variables. $A_{d}$ is a set of global linear equations. With this partitioning, it is possible to apply the LATIN method, a general computational strategy for time-dependent nonlinear problems which operates globally over the entire timespace domain. This method, described in [10], relies on some remarkable properties which are verified by most models encountered in structural mechanics. Figure 1 shows the scheme for one iteration, which consists of two steps.

\section{- The local stage at iteration $n$}

The problem consists of building $\hat{\mathbf{s}}_{n+1 / 2} \in \Gamma$ knowing $\mathbf{s}_{n} \in A_{d} \cdot\left(\hat{\mathbf{s}}_{n+1 / 2}-\mathbf{s}_{n}\right)$ must follow a search direction $\mathbf{E}^{+}$. The local step presents no difficulty. The problems to be solved are local in the space and time variables and, therefore, lend themselves to the highest degree of parallelism.

\section{- The linear stage at iteration $n$}

The problem consists of building $\mathbf{s}_{n+1} \in A_{d}$ knowing $\hat{\mathbf{s}}_{n+1 / 2} \in \Gamma .\left(\mathbf{s}_{n+1}-\hat{\mathbf{s}}_{n+1 / 2}\right)$ must follow a search direction $\mathbf{E}^{-}$.

In practise, we introduce a Lagrange multiplier $\underline{\tilde{W}}^{M}$ to ensure the admissibility of the macro forces. Then, the linear stage can be split into two parts.

- The micro problem defined on substructure $\Omega E$ and its boundary $2 \Omega E$ and on $[0, T]$

The micro problem associated with substructure $E$ can be written as: find $s_{E}$ E-admissible which 
verifies the search direction $\mathbf{E}-$. The solution to the micro problem related to substructure $\mathrm{E}$ depends only on the known quantities $\underline{f}_{d[\Omega} E, \hat{\mathbf{s}}_{E, n+1 / 2}$, on the given initial condition and on the value of $\underline{\underline{W}}_{E}^{M}$ at the boundary $\partial \Omega_{E}$ which, at this stage, is unknown. The "macro" part of the solution can be written as follows:

$$
\underline{F}_{E \mid \alpha \Omega_{E} \times[O, T]}^{M}=\mathbf{L}_{\mathbf{E}}\left(\underline{\tilde{W}}_{E \mid \partial \Omega_{E} \times[O, T]}^{M}\right)+\hat{\hat{E}}_{E, d \mid \alpha \Omega_{E} \times[O, T]}^{M}
$$

where $\hat{\underline{F}}_{E, d}^{M}$ depends on $\underline{f}_{d \mid \Omega} E, \hat{\mathbf{s}}_{E, n+1 / 2}$ and on the initial conditions. $\mathbf{L}_{\mathbf{E}}$ is a linear operator which can be interpreted as a homogenized behavior operator over the space-time substructure $\Omega_{E} \times[0, T]$. It is calculated with zero initial conditions at a relatively low cost because $\underline{W}_{E}^{M}$ on $\partial \Omega_{E} \times[0, \mathrm{~T}]$ depends on only a few scalar parameters. $\mathbf{L}_{\mathbf{E}}$ represents the coupling effect among the different scales.

The micro problems defined on $\Omega_{E} \times \mathrm{t}_{\mathrm{i}}, \mathrm{t}_{\mathrm{i}+1}[, E \in \mathbf{E}$ and $\mathrm{i} \in\{0, \ldots, \mathrm{N} \pm \mathrm{I}\}$ are independent. However, for each substructure E, their resolution must be incremental. This step lends itself to parallelism very well.

At each iteration and for each substructure, we need to solve micro problems which are evolution equations defined on $\Omega_{E} \times[0, T]$. When a precise solution is required both in space and in time, the cost of solving these problems with standard incremental methods can be prohibitive. We propose to introduce an approximation technique for the resolution of these problems, based on the concept of generalized radial functions $[9,10]$.

\section{- The macro problem defined on $\Omega \times[0, T]$}

Relation (2) and the admissibility of the "macro" forces lead to the macro problem, which is an homogenized problem on the entire space-time domain $\Omega \times[0, \mathrm{~T}]$. The macro problem yields $\underline{\tilde{W}}^{M}$ and $\underline{F}^{M}$. Then, using the micro problem again, one can determine $s_{n+1}$ completely.

Macro quantities are defined only at the interfaces; by treating the medium as a Cosserat material, one can define macrostresses, macrostrains, inside a substructure $\Omega_{E}$.

If the number of macro space-time substructures $\left.\Omega_{E} \times\right] t^{\prime} i, t^{\prime}{ }_{i+1}[$ is large, the cost of solving the macro problem can be prohibitive. Here, we also propose to build an approximation of its solution inspired from the kinematics of a Cosserat medium [6,9].

\section{Example}

Let us consider the 2D problem of a composite structure containing cracks (unilateral contact with friction with a friction parameter $\mu=0.2$ ) (Figure 2). It is fixed on the bottom and subjected to prescribed forces $\underline{F}_{1}$ and $\underline{F}_{2}$ (Figure 3). The structure is made of two types of cells, denoted I and II. The materials are viscoelastic with Young's modulus $E_{i}$, Poisson's ratio $v_{1}$ and viscosity $\eta_{\text {. }}$. The Type-II cell is homogeneous 
with a Type-1 material whose characteristics are $E_{1}=50 \mathrm{GPa}, v_{1}=0.3$ and $\eta_{1}=10 \mathrm{~s}$. The Type-ll cell consists of a matrix of Type-1 material and inclusions of Type-2 material whose characteristics are $E_{2}=250 \mathrm{GPa}$, $v_{2}=0.2$ and $\eta_{2}=1000 \mathrm{~s}$.

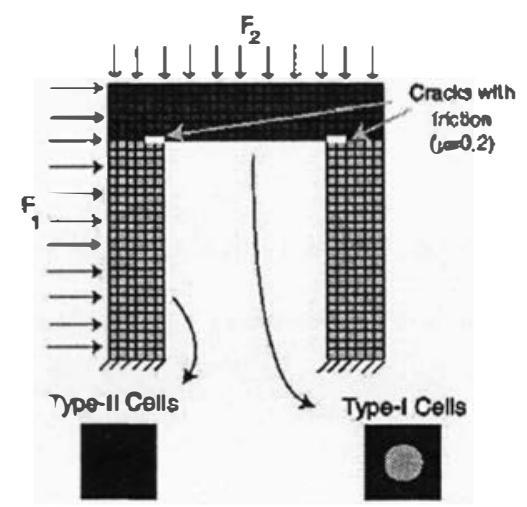

Fig. 2: Description of the problem

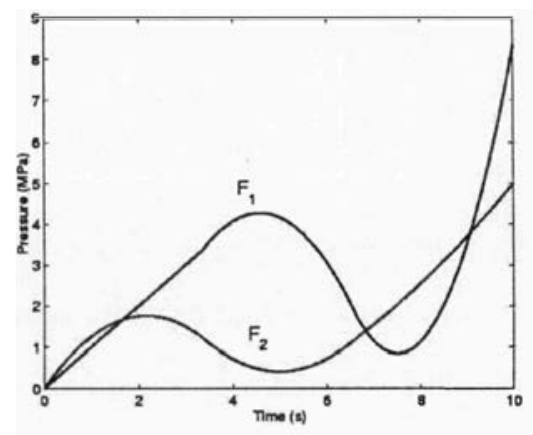

Fig. 3: Loadings $\underline{F}_{1}$ and $\underline{F}_{2}$

The time interval being considered is $[0,10 s]$ and is discretized with 60 identical micro intervals and 3 identical macro intervals. The "macro" spaces contain affine functions per interface on the space level and functions of type $z_{2}^{[0, T]}$ for the grid $\tau_{h O}$ on the time level. The "micro" spaces contain functions of type $z_{0}^{[0 . T]}$ for the grid $\tau_{h}$.

Figures 4, 5, 6, and 7 show the good agreement between macro and total forces over space and time, also in high gradient zones (in the middle of the heterogeneous zone and around the cracks). Let us remark that unilateral contact conditions are satisfied by the total quantities and satisfied in a mean sense by the macro quantities. The same results occur for displacements.

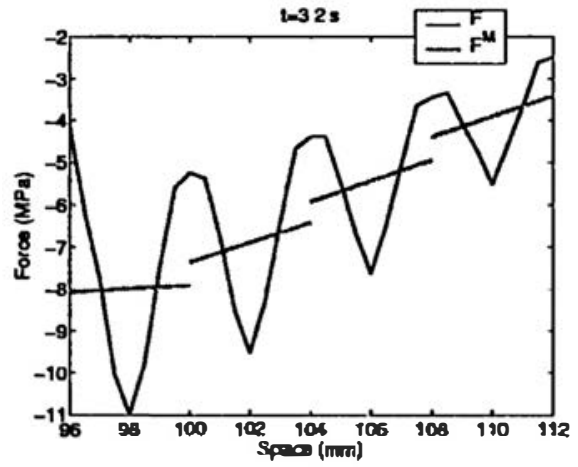

Fig. 4: Space distribution of normal component of macro and total forces on four vertical interfaces in the middle of the heterogeneous zone

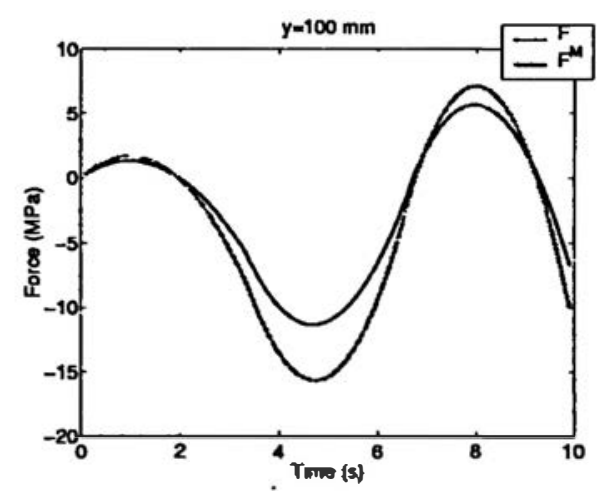

Fig. 5: Time evolution of normal component of macro and total forces on a point of an interface in the middle of the heterogeneous zone 

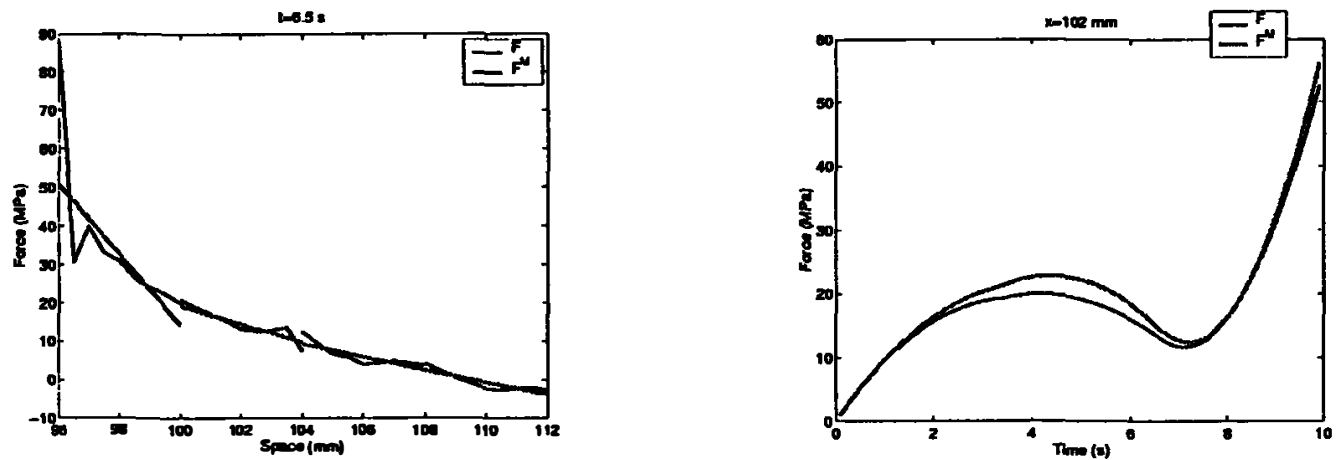

Fig. 6: Space distribution of normal component of Fig. 7: Time evolution of normal component of macro and total forces on four horizontal interfaces around the right crack macro and total forces on a point of the right crack

Figure 8 shows the evolution of the error with the number of iterations of the LATIN method. The algorithm converges towards a good solution very quickly (relative error less than 1/100 after 10 iterations).

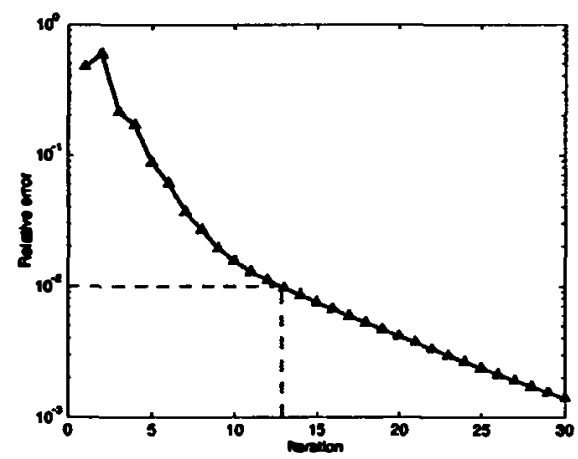

Fig. 8: Evolution with the number of iterations of an error based on interface quantities

\section{CONCLUSION}

The improved method presented here led to significant performance gains in the resolution of the linear stage. The first improvement was the introduction, for composite structures, of a third scale and the use of an approximation technique for the macro problem: this approximation is based on an analogy between the macro homogenized structure and a Cosserat medium. The second improvement was the introduction of the radial loading approximation to solve the micro problems. This strategy extends the approximation classically used in the LATIN method for quasi-static problems [7]. The gain obtained by these two improvements becomes significant as one has to deal with a large number of composite cells and needs to 
calculate a detailed solution in the time domain. The techniques described here can be used only with global methods in space and in time, which excludes classical incremental methods.

\section{REFERENCES}

[1] E. Sanchez-Palencia, Comportement local et macroscopique d'un type de mileux physiques hétérogènes, International Journal for Engineering Science, 27, 231-251 (1974)

[2] F. Devries, H. Dumontet, G. Duvaut, F. Léné, Homogenization and damage for composite structures, International Journal for Numerical Methods in Engineering, 27, 285-298 (1989)

[3] F. Feyel, J.-L. Chaboche, $F E^{2}$ multiscale approach for modelling the elasto-viscoplastic behaviour of long fiber SiC/Ti composite materials, Computer Methods in Applied Mlechanics and Engineering, 183:417-455 (2000)

[4] J.T. Oden, K. Vemaganti, N. Moes, Hierarchical modelling of heterogeneous solids, Computer Methods in Applied Mechanics and Engineering, 172, 2-25 (1999)

[5] J. Fish, K. Sheck, M. Pandheeradi, M.S. Shepard, Computational plasticity for composite structures based on mathematical homogenization : Theory and practice, Computer Methods in Applied Mechanics and Engineering, 148, 53-73 (1997)

[6] P. Ladevèze, O. Loiseau, D. Dureisseix, A micro-macro and parallel computational strategy for highly heterogeneous structures, International Journal for Numerical Methods in Engineering, 52, 121-138 (2001)

[7] P. Ladevèze, A. Nouy, A multiscale computational method with time and space homogenization, $C$. $R$. Mècanique, 330, 1-7 (2002)

[8] P. Ladevèze, Multiscale Modelling and Computational Strategies for Composites, Proceedings of the Fifth World Congress on Computational Mechanics (IVCCM V), July 7-12, 2002, Vienna, Austria, Editors: Mang, H.A.; Rammerstorfer, F.G.; Eberhardsteiner, J., Publisher: Vienna University of Technology, Austria, ISBN 3-9501554-0-6

[9] P. Ladevèze, A. Nouy, On a multiscale computational strategy with time and space homogenization for structural mechanics, Computer Methods in Applied Mechanics and Engineering, (to be published)

[10] P. Ladevèze, Nonlinear Computational Structural Mechanics - New Approaches and Non-ncremental Methods of Calculation, Springer Verlag, (1999)

[11]K. Eriksson, D. Estep, P. Hansbo, C. Johnson, Computational Differential Equations, Cambridge University Press (1996) 